${ }^{1}$ Carrera de Nutrición y Dietética, Facultad de Ciencias de la Salud. Universidad San Sebastián, Chile. ${ }^{2}$ Escuela de Nutrición y Dietética. Universidad Santo Tomás, Sede Viña del Mar. Viña del Mar, Chile. ${ }^{3}$ INTA. Universidad de Chile. aNutricionista.

bPhD en Nutrición y Alimentos.

Financiamiento: autofinanciado

Recibido el 7 de junio de 2014, aceptado el 5 de noviembre de 2014.

Correspondencia a: Samuel Durán Agüero Carrera de Nutrición y Dietética, Facultad de Ciencias de la Salud, Universidad San Sebastián Lota 2465, Providencia, Chile. samuel.duran@uss.cl

\section{Comparación del consumo de vitaminas y minerales en adultos mayores chilenos según estado nutricional}

\author{
SAMUEL DURÁN A. ${ }^{1, a, b}$, ALEJANDRA ULLOA A. ${ }^{2, a}$, \\ SUSSANNE REYES G., ${ }^{3, a, b}$
}

\section{Nutrient intake of Chilean older people according to body mass index}

\begin{abstract}
Background: An adequate consumption of micro and macro nutrients is essential to maintain an adequate health among older people. Aim: To compare the consumption of micro- and macronutrients in older people from three Chilean cities, according to their nutritional status. Material and Methods: Body mass index (BMI) was assessed and a food consumption tendency survey was applied to 976 non-disabled older people, living in the community. Thinness was defined as a BMI $<23 \mathrm{~kg} / \mathrm{m}^{2}$. Results: Twenty percent of females and $17 \%$ of males had a $B M I<23 \mathrm{~kg} / \mathrm{m}^{2}$. Participants with a higher BMI had a greater intake of micro- and macronutrients. In females, micronutrient intake was adequate among those with higher BMI, although mean intake of calcium and vitamin $B-12$ were below recommendations. In males, iron, zinc, calcium, magnesium, vitamin A, vitamin B6, vitamin B12 and pantothenic acid intake were below recommendation. Conclusions: Thin older adults, regardless of sex, had a lower intake of calories and micro- and macronutrients. Additionally, an overall low consumption of zinc, calcium, magnesium and vitamin B12 was detected.
\end{abstract}

(Rev Med Chile 2014; 142: 1594-1602)

Key words: Aged; Dietary requirements; Nutrition assessment.
L a población adulta mayor (AM) en los países en desarrollo está creciendo más rápidamente que en países desarrollados y se proyecta que para el año 2020, 70\% de las personas de más de 55 años, vivirá en países en desarrollo ${ }^{1}$. De acuerdo al último censo poblacional, para el año 2002 se estimó que $11,4 \%$ de la población chilena era $\mathrm{AM}^{2}$.

En el proceso del envejecimiento se produce un deterioro biológico resultante de la interacción de factores genéticos y ambientales, como los estilos de vida, hábitos alimentarios, actividad física y presencia de enfermedades. Se considera que el rol de los micronutrientes en esencial en este grupo etario ${ }^{3}$. Los AM presentan un requerimiento energético menor que sus pares adultos ( $<60$ años), debido a una disminución del gasto metabólico basal, cambios en la composición corporal y menor actividad física, entre otros factores.
Además, tienen mayor riesgo de malnutrición por déficit principalmente por una menor calidad de la alimentación (ingesta insuficiente de calorías o de macro y micronutrientes), o por el contrario, una mayor probabilidad de sufrir sobrepeso u obesidad por ingerir más calorías de las realmente necesarias, que muchas veces no incluyen los nutrientes adecuados, produciéndose igualmente carencias de algunos micronutrientes ${ }^{4}$.

En los AM, el principal cambio de la conducta alimentaria se expresa en la "selección de los alimentos", lo cual está influenciado por diversos factores: biológicos (señales de saciedad en función de los macronutrientes y la densidad energética del alimento), organolépticos (incluyendo textura, sabor, olor, vista), económicos, sociales (acceso a los alimentos, nivel de autonomía, nivel de educación, entorno social y apoyo familiar) y psicológicos (depresión y estrés) ${ }^{5}$. 
Una ingesta alimentaria menor a los requerimientos diarios es común en AM. Esta conducta está presente en casi $30 \%$ de las personas AM entre 70 y 80 años y que viven solos 6 .

Así, el estado nutricional del AM resulta de una compleja interacción entre la dieta y los factores socioeconómicos, físicos y psicológicos. Además, la evidencia disponible indica que la malnutrición por déficit y la obesidad limitan la capacidad de moverse, realizar las actividades diarias y empeoran las comorbilidades. Por lo tanto, se establece que el estado nutricional es un factor clave para mantener la salud y la autonomía en los $\mathrm{AM}^{7}$, aunque se desconoce si la ingesta de macro y micronutrientes es diferente según clasificación ponderal.

El principal objetivo del presente estudio es comparar la ingesta de macro y micronutrientes en AM de Valparaíso, Viña del Mar y Santiago de Chile según su estado nutricional.

\section{Materiales y Métodos}

\section{Sujetos:}

Estudio descriptivo que incluyó 976 AM de 65 años o más, autovalentes y no institucionalizados (61\% mujeres), pertenecientes a las comunas del Gran Santiago, Valparaíso y Viña del Mar. La autovalencia se determinó con el diagnóstico de funcionalidad (EFAM/Chile) ${ }^{8}$ y se consideró autovalente al AM con un puntaje $\geq 43$ puntos. Los criterios de exclusión fueron AM dependientes, con deterioro cognitivo e institucionalizados. El estudio fue aprobado por el Comité de Ética de la Universidad San Sebastián.

\section{Encuesta alimentaria}

Nutricionistas entrenados encuestaron a los AM. El instrumento sobre tendencia de consumo de alimentos semanal incluyó 31 tipos de alimentos (frutas, verduras, cereales, pan, carnes, pescados, lácteos, papas, aceites y grasas, bebidas, azúcares, etc.). Las porciones fueron descritas como utensilios típicos de uso en el hogar (vaso, taza, cuchara, cucharadita, plato, etc.). Esta información fue utilizada para calcular la ingesta diaria de nutrientes. Para ello, se utilizó el software de Evaluación de Ingesta de Alimentos del Instituto de Nutrición y Tecnología de Alimentos?. La ingesta estimada de vitaminas y minerales fue comparada con los valores de Requerimiento Promedio Estimado (EAR). El EAR es la cantidad promedio de ingesta diaria de un nutriente que logra cubrir los requerimientos de $50 \%$ de los individuos saludables, en una etapa de la vida específica ${ }^{10}$. También se comparó con la Dosis Diaria Recomendada (RDA) utilizada en el etiquetado nutricional de alimentos en Chile, basado en las directrices entregadas por el Codex y en algunos casos por la FDA y el Ministerio de Salud de Chile (MINSAL). La RDA es la cantidad promedio de ingesta diaria de nutrientes suficiente para satisfacer los requerimientos de aproximadamente todos los individuos sanos, en una etapa específica de la vida ${ }^{10}$. Además se contrastó con el Nivel Máximo de Ingesta Tolerable (UL) para vitaminas y se analizó si los valores determinados sobrepasaban a los de referencia $(\mathrm{FAO} / \mathrm{WHO} \mathrm{Hu}$ man Vitamin and Mineral Requirements 2001) ${ }^{11}$.

\section{Antropometría}

Se efectuó una evaluación antropométrica de peso y talla. El peso se determinó con un mínimo de ropa, utilizando una balanza mecánica (SECA, capacidad máxima de $220 \mathrm{~kg}$ y precisión de 50 g). La estatura se midió con un tallímetro (precisión $1 \mathrm{~mm}$ ). El estado nutricional fue determinado con el índice de masa corporal (IMC) (Peso (kg)/ talla (m) al cuadrado). Se clasificó de acuerdo a lo recomendado por el MINSAL para la evaluación del AM: $\leq 23$ delgadez o enflaquecido (EF); $>23$ a $<28$ normal; $\geq 28$ a $<32$ sobrepeso (SP) y $\geq 32$ obesidad $(\mathrm{OB})^{12}$.

\section{Análisis estadístico}

Para la evaluación entre grupos de utilizó el análisis de varianza (ANOVA) y para la comparación por pares se utilizó el test de Bonferroni. El consumo promedio de vitaminas y minerales se comparó con el EAR, la RDA y el UL (para hombres y mujeres). Para el análisis estadístico se utilizó el programa SPSS versión 19.0, considerando significativo un valor de $\mathrm{p}<0,05$.

\section{Resultados}

Por diseño las diferencias en peso e IMC se presentan en todos los grupos de AM, 20,1\% de las mujeres y $16,9 \%$ de los hombres estaban enflaquecidos (Tabla 1).

Para las mujeres AM, a mayor IMC mayor es el consumo de macro y micronutrientes. Las mujeres enflaquecidas al compararlas con mujeres obesas, consumen significativamente menos 
calorías $(\mathrm{p}<0,05)$, proteínas $(\mathrm{p}<0,05)$, lípidos $(\mathrm{p}<0,05)$, hierro $(\mathrm{p}<0,05)$, zinc $(\mathrm{p}<0,05)$, selenio $(\mathrm{p}<0,05)$, vitamina-A $(\mathrm{p}<0,05)$, vitamina-B2 $(\mathrm{p}<0,05)$, vitamina-B3 $(\mathrm{p}<0,05)$, vitamina-B6 $(\mathrm{p}<0,05)$, vitamina-B12 $(\mathrm{p}<0,05)$, ácido pantoténico $(\mathrm{p}<0,05)$ y vitamina-C $(\mathrm{p}<0,05)($ Tabla 2$)$.
En el caso de los hombres, al igual que las mujeres, a menor IMC menor es el consumo de macro y micronutrientes. Los hombres enflaquecidos al compararlos con los obesos, presentan una menor ingesta de calorías $(\mathrm{p}<0,05)$, proteínas $(\mathrm{p}<0,05)$, hierro $(\mathrm{p}<0,05)$, zinc $(\mathrm{p}<0,05)$, selenio

Tabla 1. Comparación en variables de edad y antropometría según estado nutricional

\begin{tabular}{|c|c|c|c|c|}
\hline & Enflaquecido & Normal & SP & OB \\
\hline Mujeres & $n=100$ & $n=244$ & $n=159$ & $n=94$ \\
\hline Edad (años) & $72,0 \pm 9,1^{a}$ & $69,3 \pm 6,6^{a}$ & $70,9 \pm 7,4$ & $70,6 \pm 7,7$ \\
\hline Peso (k) & $55,0 \pm 6,1^{a, b, c}$ & $63,7 \pm 6,2^{\mathrm{a}, \mathrm{d}, \mathrm{e}}$ & $72,8 \pm 6,5^{b, d, f}$ & $83,6 \pm 10,1^{c, e, f}$ \\
\hline Talla (m) & $1,59 \pm 0,05^{a, b, d}$ & $1,58 \pm 0,06^{c}$ & $1,56 \pm 0,06^{\mathrm{a}, \mathrm{d}, \mathrm{e}}$ & $1,53 \pm 0,06^{b, c, e}$ \\
\hline $\mathrm{IMC}\left(\mathrm{k} / \mathrm{m}^{2}\right)$ & $21,5 \pm 1,2^{a, b, c}$ & $25,4 \pm 1,3^{\mathrm{a}, \mathrm{d}, \mathrm{e}}$ & $29,5 \pm 1,0^{b, d, f}$ & $35,4 \pm 3,5^{c, e, f}$ \\
\hline Hombres & $n=64$ & $n=203$ & $n=81$ & $n=30$ \\
\hline Edad (años) & $72,9 \pm 7,9$ & $70,6 \pm 6,7$ & $70,6 \pm 7,7$ & $72,5 \pm 7,1$ \\
\hline Peso (k) & $62,6 \pm 6,7^{a, b, c}$ & $72,7 \pm 7,0^{\mathrm{a}, \mathrm{d}, \mathrm{e}}$ & $82,0 \pm 7,9^{b, d, f}$ & $91,7 \pm 10,6^{c, e, f}$ \\
\hline Talla (m) & $1,68 \pm 0,07^{a}$ & $1,68 \pm 0,07^{b}$ & $1,66 \pm 0,07$ & $1,62 \pm 0,06^{a, b}$ \\
\hline $\mathrm{IMC}\left(\mathrm{k} / \mathrm{m}^{2}\right)$ & $21,8 \pm 1,2^{a, b, c}$ & $25,3 \pm 1,3^{a, d, e}$ & $29,7 \pm 1,17^{b, d, f}$ & $34,6 \pm 2,6 c, e, f$ \\
\hline
\end{tabular}

Valores expresados en media $\pm \mathrm{DE}$. Los valores medios en una fila con la misma letra fueron significativamente diferentes $(p<0,05)$. Prueba Anova. Post hoc Bonferroni.

Tabla 2. Comparación entre la ingesta de macro y micronutrientes según estado nutricional en adultos mayores mujeres

\begin{tabular}{|c|c|c|c|c|}
\hline & $\begin{array}{l}\text { Enflaquecido } \\
\qquad n=100\end{array}$ & $\begin{array}{l}\text { Normal } \\
n=244\end{array}$ & $\begin{array}{c}\text { Sobrepeso } \\
n=159\end{array}$ & $\begin{array}{l}\text { Obeso } \\
n=94\end{array}$ \\
\hline Calorías (Kcal) & $1.511,2 \pm 416,5^{a, b, c}$ & $1.666,3 \pm 462,4^{c}$ & $1.820,3 \pm 520,2^{b}$ & $1.963,6 \pm 649,2^{a}$ \\
\hline Proteínas (g) & $62,0 \pm 22,8^{a, d}$ & $68,6 \pm 25,9^{b}$ & $74,2 \pm 24,9^{c, d}$ & $84,3 \pm 27,9^{a, b, c}$ \\
\hline Lípidos (g) & $42,0 \pm 18,3^{a, c}$ & $48,3 \pm 19,5^{b, d}$ & $56,1 \pm 23,9^{c, d}$ & $55,8 \pm 22,8^{a, b}$ \\
\hline Carbohidratos (g) & $234,8 \pm 79,7^{a}$ & $242,0 \pm 83,2^{b}$ & $255,4 \pm 81,0$ & $282,8 \pm 116,4^{a, b}$ \\
\hline Fibra Dietética (g) & $19,9 \pm 10,4$ & $20,1 \pm 7,3$ & $22,0 \pm 8,0$ & $25,9 \pm 10,1$ \\
\hline Hierro (mg) & $10,5 \pm 4,4^{a, b}$ & $11,6 \pm 3,9^{c, d}$ & $12,6 \pm 4,5^{\mathrm{a}, \mathrm{c}, \mathrm{e}}$ & $14,9 \pm 6,1^{b, d, e}$ \\
\hline Zinc (mg) & $5,1 \pm 2,3^{a, b, c}$ & $5,9 \pm 2,4^{\mathrm{a}, \mathrm{d}, \mathrm{e}}$ & $6,7 \pm 2,8^{b, d, f}$ & $7,7 \pm 3,2^{c, e, f}$ \\
\hline Calcio (mg) & $653,6 \pm 376,0$ & $663,9 \pm 329,0$ & $743,3 \pm 456,9$ & $787,5 \pm 454,3$ \\
\hline Cobre (mg) & $1,3 \pm 6,1$ & $1,7 \pm 7,8$ & $1,2 \pm 4,9$ & $0,9 \pm 3,9$ \\
\hline Magnesio (mg) & $119,8 \pm 66,8^{a, b}$ & $130,0 \pm 69,6^{c}$ & $145,8 \pm 76,9^{a}$ & $177,2 \pm 92,0^{b, c}$ \\
\hline Selenio $(\mu \mathrm{g})$ & $66,3 \pm 34,9^{a, b}$ & $75,0 \pm 39,8$ & $80,6 \pm 35,1^{c}$ & $85,4 \pm 38,7^{b}$ \\
\hline Vitamina A ( $\mu \mathrm{g}$ ER) & $289,7 \pm 421^{a, b}$ & $381,0 \pm 482,0$ & $494,3 \pm 514,6^{a}$ & $610 \pm 694,3^{b}$ \\
\hline B1 (mg) & $1,4 \pm 1,3$ & $1,4 \pm 0,4^{\mathrm{a}}$ & $1,5 \pm 0,5$ & $1,7 \pm 0,6^{a}$ \\
\hline B2 (mg) & $1,0 \pm 0,5^{a, b}$ & $1,1 \pm 0,8$ & $1,3 \pm 0,9^{a}$ & $1,3 \pm 0,5^{b}$ \\
\hline B3 (ENs) & $11,9 \pm 3,9^{a, b}$ & $12,6 \pm 3,7^{c, d}$ & $13,9 \pm 4,4^{\mathrm{a}, \mathrm{c}, \mathrm{e}}$ & $15,8 \pm 5,2^{b, d, e}$ \\
\hline B6 (mg) & $0,90 \pm 0,3^{a, b}$ & $0,97 \pm 0,4^{c}$ & $1,0 \pm 0,4^{a, d}$ & $1,2 \pm 0,5^{b, c, d}$ \\
\hline Folatos $(\mu \mathrm{g})$ & $505,7 \pm 271,7$ & $529,6 \pm 239,3$ & $579,1 \pm 246,1$ & $682,9 \pm 316,0$ \\
\hline B12 $(\mu \mathrm{g})$ & $0,92 \pm 0,4^{a, b}$ & $1,04 \pm 0,6^{c}$ & $1,17 \pm 0,6^{a}$ & $1,30 \pm 0,8^{b, c}$ \\
\hline Ac. Pantoténico (mg) & $3,05 \pm 1,2^{a}$ & $3,09 \pm 1,4^{b}$ & $3,03 \pm 1,1^{c}$ & $3,82 \pm 1,2^{a, b, c}$ \\
\hline Vitamina C (mg) & $51,7 \pm 45,9^{a}$ & $53,0 \pm 38,4^{b}$ & $60,0 \pm 45,5$ & $75,1 \pm 65,6^{a, b}$ \\
\hline
\end{tabular}

Valores expresados en media \pm DE. Los valores medios en una fila con la misma letra fueron significativamente diferentes $(p<0,05)$. Prueba Anova. Post hoc Bonferroni. 
( $p<0,05)$, vitamina-B1 $(\mathrm{p}<0,05)$, vitamina-B3 $(\mathrm{p}<0,05)$, vitamina-B6 $(\mathrm{p}<0,05)$, vitamina-B12 $(\mathrm{p}<0,05)$ y ácido pantoténico $(\mathrm{p}<0,05)$ (Tabla 3$)$.

Por kilo de peso, se observa una mayor ingesta de calorías y de macronutrientes tanto en mujeres como en hombres enflaquecidos (Tabla 4).
Por otra parte y en el caso de las mujeres, las adecuaciones para calcio, magnesio, vitamina-A y vitamina-B12, se encuentran en todos los grupos bajo los EAR. Por debajo de las RDA, se encuentran el hierro, zinc, calcio, magnesio, vitamina-A, vitamina-B6 y vitamina-B12. En el caso de los

Tabla 3. Comparación entre la ingesta de macro y micronutrientes según estado nutricional en adultos mayores hombres

\begin{tabular}{|c|c|c|c|c|}
\hline & $\begin{array}{l}\text { Enflaquecido } \\
\qquad n=64\end{array}$ & $\begin{array}{l}\text { Normal } \\
n=204\end{array}$ & $\begin{array}{c}\text { Sobrepeso } \\
n=81\end{array}$ & $\begin{array}{l}\text { Obeso } \\
n=30\end{array}$ \\
\hline Calorías & $1.698,8 \pm 482,8^{a, b, c}$ & $1.920,2 \pm 513,2^{a}$ & $2.069,3 \pm 654,0^{b}$ & $2.020,5 \pm 505,7^{c}$ \\
\hline Proteínas (g) & $69,5 \pm 28,3^{a, b}$ & $78,3 \pm 27,8$ & $82,2 \pm 28,9^{a}$ & $90,4 \pm 30,7^{b}$ \\
\hline Lípidos (g) & $50,0 \pm 20,2$ & $58,4 \pm 23,7$ & $58,2 \pm 23,5$ & $57,7 \pm 22,2$ \\
\hline Carbohidratos (g) & $243,5 \pm 82,2^{a}$ & $274,5 \pm 87,8^{b}$ & $312,7 \pm 112,5^{a, b}$ & $287,2 \pm 66,6$ \\
\hline Fibra Dietética (g) & $19,8 \pm 10,8^{a}$ & $22,4 \pm 7,7^{b}$ & $26,3 \pm 13,2^{a, b}$ & $25,6 \pm 12,1$ \\
\hline Hierro (mg) & $11,8 \pm 4,8^{a, b}$ & $13,4 \pm 4,6^{b}$ & $15,7 \pm 6,5^{b}$ & $15,7 \pm 5,6^{a}$ \\
\hline Zinc (mg) & $6,0 \pm 2,9^{a, b}$ & $7,0 \pm 2,6$ & $7,3 \pm 3,0^{b}$ & $8,4 \pm 3,1^{\mathrm{a}}$ \\
\hline Calcio (mg) & $683,6 \pm 437,7$ & $736,9 \pm 396,8$ & $743,6 \pm 381,3$ & $822,7 \pm 606,4$ \\
\hline Cobre (mg) & $1,6 \pm 7,7$ & $1,7 \pm 7,4$ & $1,0 \pm 0,5$ & $0,9 \pm 0,3$ \\
\hline Magnesio (mg) & $137,8 \pm 92,6$ & $149,6 \pm 70,1$ & $167,3 \pm 122,3$ & $192,4 \pm 131,6$ \\
\hline Selenio $(\mu \mathrm{g})$ & $76,3 \pm 42,2^{a, b}$ & $87,2 \pm 39,1$ & $100,4 \pm 41,5^{a}$ & $103,5 \pm 35,0^{b}$ \\
\hline Vitamina A ( $\mu \mathrm{g} E R)$ & $415,7 \pm 753,0$ & $378,4 \pm 319,0$ & $471,7 \pm 696,1$ & $486,2 \pm 524,9$ \\
\hline B1 (mg) & $1,4 \pm 0,5^{a, b, c}$ & $1,7 \pm 0,5^{a, d}$ & $1,9 \pm 0,7^{b, d}$ & $1,8 \pm 0,4^{c}$ \\
\hline B2 (mg) & $1,2 \pm 0,8$ & $1,4 \pm 0,9$ & $1,4 \pm 0,6$ & $1,5 \pm 0,9$ \\
\hline B3 (ENs) & $12,7 \pm 4,3^{a, b, d}$ & $14,9 \pm 4,5^{c, d}$ & $16,8 \pm 5,8^{b, c}$ & $17,0 \pm 3,7^{a}$ \\
\hline B6 (mg) & $0,94 \pm 0,4^{\mathrm{a}, \mathrm{b}}$ & $1,11 \pm 0,4$ & $1,19 \pm 0,6^{a}$ & $1,29 \pm 0,5^{b}$ \\
\hline Folatos $(\mu \mathrm{g})$ & $531,9 \pm 259,6$ & $621,8 \pm 254,7$ & $766,6 \pm 378,5$ & $713,7 \pm 258,6$ \\
\hline B12 $(\mu \mathrm{g})$ & $1,0 \pm 0,6^{a}$ & $1,2 \pm 0,7$ & $1,3 \pm 0,9$ & $1,5 \pm 1,0^{a}$ \\
\hline Ac. Pantoténico (mg) & $3,1 \pm 1,2^{a, b}$ & $3,5 \pm 1,2$ & $3,9 \pm 1,7^{a}$ & $4,0 \pm 1,3^{b}$ \\
\hline Vitamina C (mg) & $44,1 \pm 50,8$ & $55,2 \pm 36,8$ & $55,6 \pm 45,2$ & $58,6 \pm 51,0$ \\
\hline
\end{tabular}

Valores expresados en media \pm DE. Los valores medios en una fila con la misma letra fueron significativamente diferentes $(p<0,05)$. Prueba Anova. Post hoc Bonferroni.

Tabla 4. Comparación de calorías y macronutrientes por kilogramo de peso en adultos mayores

\begin{tabular}{|c|c|c|c|c|}
\hline & Enflaquecido & Normopeso & Sobrepeso & Obesidad \\
\hline \multicolumn{5}{|l|}{ Mujeres } \\
\hline Kcal & $31,8 \pm 9,7^{a, b, c}$ & $28,2 \pm 8,5^{\mathrm{a}, \mathrm{d}, \mathrm{e}}$ & $25,7 \pm 7,7^{b, d}$ & $23,6 \pm 7,5^{c}$ \\
\hline Proteínas (g) & $1,2 \pm 0,4^{a, b, c}$ & $1,1 \pm 0,4^{\text {a,d.e }}$ & $1,0 \pm 0,3^{b . d}$ & $0,9 \pm 0,3^{c . e}$ \\
\hline Lípidos (g) & $0,9 \pm 0,3^{a, b, c}$ & $0,8 \pm 0,3^{a, d}$ & $0,8 \pm 0,4^{b, e}$ & $0,6 \pm 0,2^{c, d, e}$ \\
\hline Carbohidratos (g) & $4,5 \pm 1,5^{a, b, c}$ & $4,0 \pm 1,1^{\mathrm{a}, \mathrm{d}, \mathrm{e}}$ & $3,5 \pm 1,1^{b, d}$ & $3,3 \pm 1,3^{c, e}$ \\
\hline \multicolumn{5}{|l|}{ Hombres } \\
\hline Kcal & $30,5 \pm 8,7^{a, b, c}$ & $27,6 \pm 7,4^{\mathrm{a}, \mathrm{d}, \mathrm{e}}$ & $25,7 \pm 7,3^{b, d}$ & $22,5 \pm 6,1^{c, e}$ \\
\hline Proteínas (g) & $1,2 \pm 0,4^{a, b, c}$ & $1,1 \pm 0,3^{a}$ & $1,0 \pm 3,4^{b}$ & $0,9 \pm 0,3^{c}$ \\
\hline Lípidos (g) & $0,8 \pm 0,3^{a, b}$ & $0,8 \pm 0,3^{c}$ & $0,7 \pm 0,2^{a}$ & $0,6 \pm 0,2^{b . c}$ \\
\hline Carbohidratos (g) & $4,3 \pm 1,4^{a, b, c}$ & $3,9 \pm 1,2^{a . d}$ & $3,7 \pm 1,1^{b, e}$ & $3,0 \pm 0,8^{\text {c,dee }}$ \\
\hline
\end{tabular}

Valores expresados en media $\pm \mathrm{DE}$. Los valores medios en una fila con la misma letra fueron significativamente diferentes $*<0,05$. Prueba Anova. Post hoc Bonferroni. 


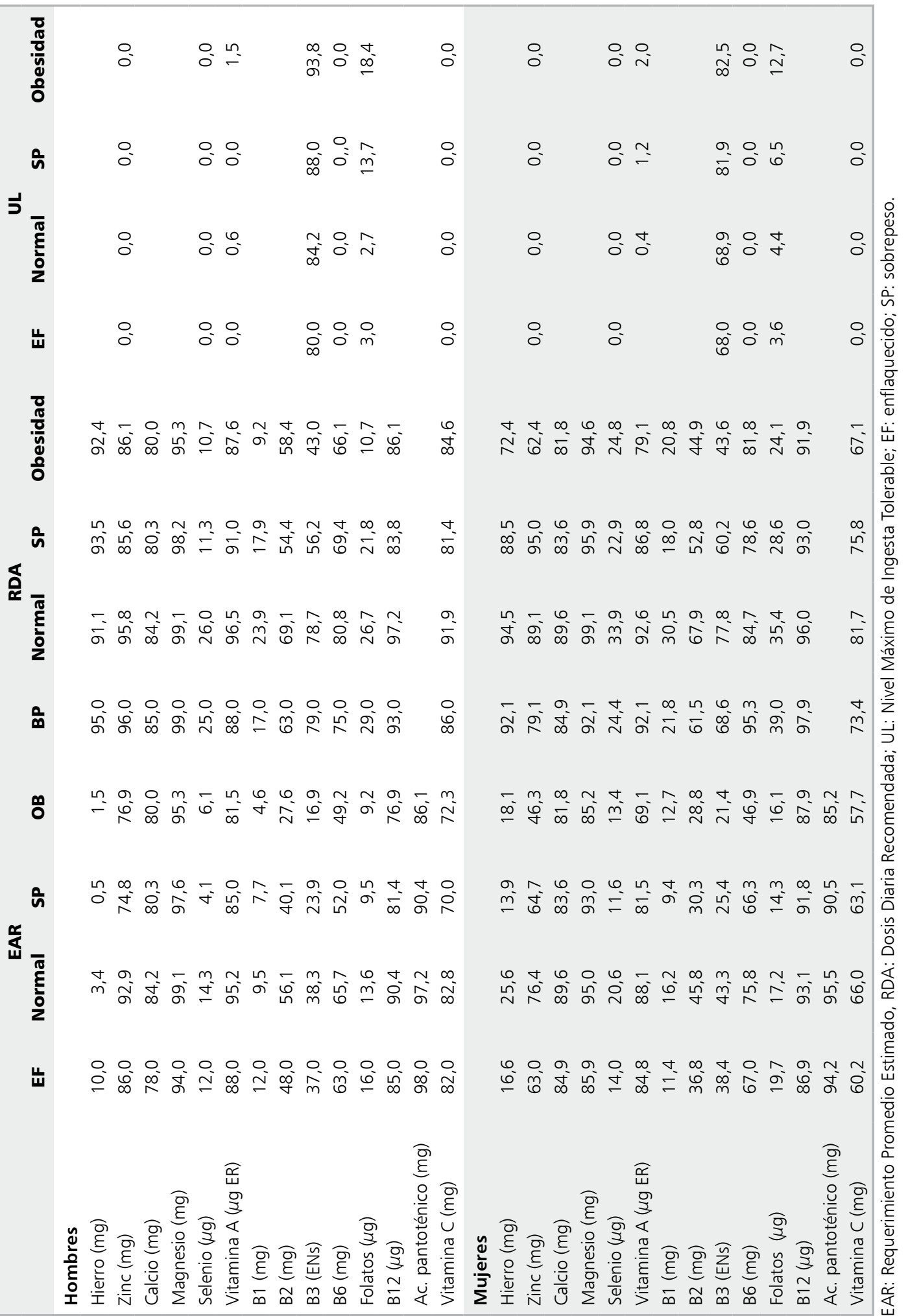


hombres y en todos los grupos, el zinc, calcio, magnesio y vitamina-A, vitamina-B12, ácido pantoténico y vitamina-C se encuentran bajo los EAR. El hierro, zinc, calcio, magnesio, vitamina$A$, vitamina-B12 y vitamina-C se encuentran bajo las RDA. Tanto para los hombres como para las mujeres, el porcentaje de AM sobre la UL de vitamina-B3 es elevado (Tabla 5).

\section{Discusión}

Los principales resultados obtenidos fueron que los AM enflaquecidos, independientemente del sexo, consumen menor cantidad de calorías, macro y micronutrientes, al compararlos con los otros grupos. Al corregir la ingesta por kilo de peso, son los AM enflaquecidos quienes presentan las mayores ingestas de calorías y macronutrientes. Además se observa que independiente del estado nutricional y de acuerdo a la RDA, existen bajas ingestas de hierro, calcio, magnesio, vitamina-A $\mathrm{y}$ vitamina-B12.

En nuestro estudio, los nutrientes en los cuales más de $80 \%$ de los AM presentaron ingesta inferiores al EAR fueron el zinc, calcio, magnesio, ácido pantoténico, vitamina-A, vitamina- $\mathrm{C}$ y vitamina-B12. Los nutrientes en situación de riesgo son similares a los encontrados en población AM europea $^{13}$. En los Estados Unidos de Norteamérica, los datos del NHANES III revelan que entre los adultos y AM $20 \%$ de las mujeres y $7 \%$ de los hombres tienen una ingesta por debajo del EAR ${ }^{14}$. Los datos del NHANES 2001-2002 indican que 31\%, $12 \%$ y $8 \%$ de la población consume cantidades por debajo del EAR de vitamina-C, zinc y ácido fólico, respectivamente ${ }^{15}$. En cambio, la encuesta de Canadian Community Health Survey demostró que entre 10-35\% de la población AM consumía menos del EAR para ácido fólico, vitamina-B6 y zinc ${ }^{16}$.

Es interesante destacar que los AM obesos presentan las mayores ingestas de vitaminas y minerales, es probable que esta mayor ingesta, especialmente hierro, se deba a mayor consumo de pan. En Chile, el pan está fortificado con las vitaminas B1, B2, B3, ácido fólico y hierro. Un reciente estudio realizado en universitarios chilenos muestran que el pan aporta 35\%, 33\%, 39\%, $67 \%$ y $34 \%$ de la ingesta total de vitaminas B1, B2, $\mathrm{B} 3$, ácido fólico y hierro, respectivamente ${ }^{17}$. Por lo tanto y debido al posible menor consumo de pan, los AM enflaquecidos tendrían menor ingesta de estos micronutrientes.

Con respecto a las proteínas, en ambos sexos la ingesta era menor en el grupo enflaquecido comparado con los grupos sobrepeso y obesos. Al ajustar por $\mathrm{kg}$ de peso, las ingestas son similares entre los grupos. Recientemente se ha sugerido como un factor adicional para evitar la sarcopenia, una ingesta de 1,2 a 1,5 g proteínas $/ \mathrm{kg} /$ peso $^{18}$, aunque lo resultados aún son contradictorios ${ }^{19}$. Nuestros resultados indican que ninguno de los grupos alcanza esta recomendación. Un estudio prospectivo informó que el consumo de proteína animal se correlacionaba positivamente con la densidad mineral ósea en mujeres ${ }^{20}$. Por otra parte, otros estudios reportan que una dieta alta en proteínas podría tener un efecto nocivo sobre la salud ósea debido a alteraciones en el metabolismo ácido-base, producido por el catabolismo de las proteínas, forzando posiblemente al tejido óseo a actuar como amortiguador, lo que resulta en la salida del calcio óseo ${ }^{21,22}$. Finalmente, un reciente estudio reportó que una mayor ingesta proteica (>20\% de las proteínas expresadas en base a kcal/ totales en hombres/mujeres) presentó una menor probabilidad de una baja densidad mineral ósea ${ }^{23}$. En nuestros sujetos, el porcentaje de calorías aportado por las proteínas fue de $16,4 \%$ en mujeres enflaquecidas, normopeso y sobrepeso y de $17,1 \%$ en mujeres obesas, en cambio en hombres fue de $16,3 \%$ en enflaquecidos y normopeso, $15,8 \%$ en sobrepeso y $17,9 \%$ en obesos.

Recientemente, una investigación realizada en AM chilenos ${ }^{24}$ determinó una elevada prevalencia de deficiencia de zinc sérico, que alcanzó a 66,8\% de la población. La disminución de la ingesta es la principal etiología de la deficiencia de este mineral ${ }^{25,26}$. Se estima que su deficiencia es un problema prevalente en los países en desarrollo y que su magnitud no sería muy distinta de la deficiencia de hierro ${ }^{27}$. Se ha demostrado que $29 \%$ de los residentes de hogares de ancianos presentan bajas concentraciones de zinc sérico $(<70 \mathrm{mg} / \mathrm{dl})$, a pesar de ser suplementados con $7 \mathrm{mg} /$ día de zinc (como sulfato) durante un año, además se determinó que la mortalidad por todas las causas fue $39 \%$ menor en pacientes con $\geq 70 \mathrm{mg}$ zinc sérico/ $\mathrm{dl}^{28}$. El zinc podría ser un marcador bioquímico de una baja ingesta proteica, sugiriendo que puede jugar un papel crucial en todas las causas de mortalidad en los ancianos. Nuestros resultados 
indicaron que un gran porcentaje de AM de ambos sexos, tienen una ingesta inadecuada de zinc, en especial los AM enflaquecidos.

Una de las principales causas de discapacidad en las personas mayores es la osteoporosis. Entre los factores de riesgo conocidos podemos nombrar la baja ingesta de calcio y vitamina- $D$, ambas protegen contra la osteoporosis y reducen la pérdida ósea en los últimos años de vida ${ }^{15}$. En nuestro estudio se observa que la adecuación dietaria para este mineral alcanza $65 \%$ en mujeres y $68 \%$ en hombres, resultado similar a otro realizado en $\mathrm{AM}$ chilenos $^{29}$. Estudios previos indican que la ingesta de calcio proveniente de los alimentos es inversamente proporcional a la regulación de la presión arterial $^{30}$. También se sugiere una relación inversa entre productos lácteos y resistencia a la insulina y diabetes tipo $2^{31}$.

La vitamina-A (retinol) juega un papel esencial en el sistema inmunológico, su deficiencia se asocia a una exacerbación de la inmunodeficiencia ${ }^{32}$, un reducido y/o un desbalance del conteo de linfocitos ${ }^{33} y$ una desregulación en la producción de anticuerpos ${ }^{34}$. De acuerdo a nuestros resultados, los AM presentaron bajas ingestas de este micronutriente.

En este estudio, tanto los hombres como las mujeres presentaron una baja ingesta de vitaminaB12, en especial los AM enflaquecidos. Situación contraria se observa con la ingesta de ácido fólico, donde en todos los grupos se supera $100 \%$ de la recomendación. La vitamina-B12 es un micronutriente esencial, ejerce un rol en la maduración de los eritrocitos que participan en las vías metabólicas necesarias para la función del sistema nervioso. Además, desempeña un rol clave en el metabolismo de un carbono (transferencia de un grupo metilo), necesario para la síntesis de neurotransmisores, colina, nucleótidos y fosfolípidos ${ }^{35}$. Bajos niveles de vitamina-B12 y folato y elevadas concentraciones de homocisteína se han asociado en muchos estudios con la densidad mineral ósea y el incremento en el riesgo de fracturas en $\mathrm{AM}^{36,37}$. Además, niveles aumentados de homocisteína junto a niveles bajos de vitamina-B12, ácido fólico y B6, se correlacionan con la diminución cognitiva, neuropatía periférica y trastornos psiquiátricos ${ }^{38}$. Junto con la edad, la atrofia gástrica, la baja acidez y la menor producción del factor intrínseco, resulta en una menor absorción de la vitamina-B12 ${ }^{39}$. Adicionalmente, el nivel socioeconómico bajo, la infección por Helicobacter pylori y una dieta vegetariana podrían incrementar la deficiencia de la vitamina-B12 $2^{40}$.

En Chile, desde el año 2005, los AM beneficiarios de Programa de Alimentación Complementaria del Adulto Mayor (PACAM) reciben la vitamina-B12 a través de alimentos fortificados que les proporciona aproximadamente $1,7 \mathrm{mg} /$ día, lo que equivale a $71 \%$ de la $\mathrm{RDA}^{35}$. Es importante destacar que en el presente estudio, se excluyeron los alimentos entregados por este programa. Tomando en cuenta que desconoce la ingesta actual de este alimento en la población AM, es de esperar que consumiendo estos productos complementarios los AM presenten mayores niveles de ingesta que los vistos en este estudio.

Finalmente, entre las fortalezas del estudio podemos nombrar el elevado número de AM encuestados, ya que los estudios sobre este tema son escasos. Entre las limitaciones podemos mencionar que es un estudio transversal y no es posible inferir relaciones de causalidad.

\section{Conclusión}

El principal hallazgo del presente estudio es que la ingesta de nutrientes al compararla según estado nutricional es significativamente inferior en AM enflaquecidos de ambos sexos, lo que perpetua este estado nutricional y aumenta el riesgo de deficiencia de vitaminas y minerales, afectando finalmente la salud y calidad de vida de este grupo etario cada día más importante en nuestro país. Además, es importante destacar al PACAM como un gran aporte en la ingesta de micronutrientes. Por lo tanto, la cobertura debería ser mayor y al igual que el monitoreo periódico.

Agradecimientos: A los estudiantes de nutrición y dietética, Luis Gamboa Henríquez, Claudio Cariman Berríos, Sara Romero, Scarlet Vildoso, Carolina Araneda Contreras, Karen Cerda, Katherine Callejas, Hernán von Martens.

\section{Referencias}

1. Zohoori N. Nutrition and healthy functioning in the developing world. J Nut 2001; 131 (9): 2429S-32S.

2. Instituto Nacional de Estadísticas de Chile (INE): Dis- 
ponible: http://www.ine.cl/canales/chile_estadistico/ estadisticas_sociales_culturales/adultosmayores/adultos_mayores.php

3. Berry E. Chronic Disease: How can Nutrition Moderate the Effects? Nutrition Reviews 1994; 52: S28-S30.

4. San Mauro I, Cendón M, Soulas C, Rodríguez D. Grupo de Investigación NIPAH Nutrición en Inmigración, Pobreza y Ayuda Humanitaria. Meal planning in the elderly: nutritional and economic aspects. Nutr Hosp 2012; 27 (6): 2116-221.

5. Payette H, Shatenstein B. Determinants of healthy eating in community-dwelling elderly people. Can J Public Health 2005; 96: S27-35.

6. van Staveren WA, de Groot LC, Burema J, de Graaf C. Energy balance and health in SENECA participants. Survey in Europe on Nutrition and the Elderly, a Concerted Action. Proc Nutr Soc1995; 54: 617-29.

7. Locher JL, Ritchie CS, Robinson CO, Roth DL, Smith West D, Burgio KL. A multidimensional approach to understanding under-eating in homebound older adults: the importance of social factors. Gerontologist 2008; 48 (2): 223-34.

8. Manual de Aplicación del Examen de Medicina Preventiva del Adulto Mayor. (Chile) disponible en: http://www.minsal.cl/portal/url/item/ablf81f43ef0c2a6e04001011e011907.pdf

9. Programa de Evaluación de Ingesta de Alimentos, INTA, Universidad de Chile, 2006.

10. García A. Ingesta de Nutrientes: Conceptos y Recomendaciones internacionales (1 $1^{\text {a Parte }) . ~ N u t r ~ H o s p ~ 2006 ; ~} 21$ (4): 291-9.

11. FAO/WHO. Human Vitamin and Mineral Requirements.2001. Disponible en: http://www.fao.org/docrep/004/y2809e/y2809e0d.htm\#bm13.

12. Orientación técnica para la atención de salud de las personas adultas mayores en atención primaria. Ministerio de Salud. Gobierno de Chile. http://web.minsal.cl/sites/ default/files/files/ot_a_MAYOR.pdf

13. Román Viñas B, Ribas Barba L, Ngo J, Gurinovic M, Novakovic R, Cavelaars A, et al. Projected prevalence of inadequate nutrient intakes in Europe. Ann Nutr Metab 2011; 59 (2-4): 84-95.

14. Bailey RL, Dodd KW, Gahche JJ, Dwyer JT, McDowell MA, Yetley EA, et al. Total folate and folic acid intake from foods and dietary supplements in the United States: 2003-2006. Am J Clin Nutr 2010; 91: 231-7.

15. Moshfegh A, Goldman J, Cleveland L. What we eat in America-NANHES 2001-2002: usual nutrient intakes from food compared to dietary references intake. Washington, US Department of Agriculture, Agricultural Research Service, 2005.
16. Health Canada, Statistics Canada: Canadian Community Health Survey-cycle 2.2, nutrition (2004): nutrient intakes from food-provincial, regional and national data. Ottawa, Health Canadá, 2009.

17. Durán S, Reyes S, Gaete MC. Aporte de vitaminas y minerales por grupo de alimentos en estudiantes universitarios chilenos. Nutr Hosp 2013; 28 (3): 830-8.

18. Morley JE. Sarcopenia in the elderly. Fam Pract 2012; 29: 144-8.

19. Alemán-Mateo H, Macías L, Esparza-Romero J, Astiazaran-García H, Blancas AL. Physiological effects beyond the significant gain in muscle mass in sarcopenic elderly men: evidence from a randomized clinical trial using a protein-rich food. Clin Interv Aging 2012; 7: 225-34.

20. Cao JJ, Nielsen FH. Acid diet (high-meat protein) effects on calcium metabolism and bone health. Curr Opin Clin Nutr Metab Care 2010; 13: 698-702.

21. Promislow JH, Goodman-Gruen D, Slymen DJ, BarrettConnor E. Protein consumption and bone mineral density in the elderly: the Rancho Bernardo Study. Am J Epidemiol 2002; 155: 636-44.

22. Barzel US. The skeleton as an ion exchange system: implications for the role of acid-base imbalance in the genesis of osteoporosis. J Bone Miner Res 1995; 10: 1431-6.

23. Kim J, Kim B, Lee H, Choi H, Won C. The Relationship between Prevalence of Osteoporosis and Proportion of Daily Protein Intake. Korean J Fam Med 2013; 34 (1): 43-8.

24. Olivares M, Lera L, Albala C, Pizarro F, Araya M. Prevalencia de las deficiencias de zinc y cobre en adultos mayores de la Región Metropolitana de Santiago. Rev Med Chile 2011; 139: 283-9.

25. Olivares M, Pizarro F, De Pablo S, Araya M, Uauy R. Iron, zinc and copper: contents in common Chilean foods and daily intakes in Santiago City, Chile. Nutrition 2004; 20: 205-12.

26. Sharkey JR, Branch LG, Zohoori N, Giuliani C, BusbyWhitehead J, Haines PS. Inadequate nutrient intakes among homebound elderly and their correlation with individual characteristics and health-related factors. Am J Clin Nutr 2002; 76: 1435-45.

27. International Zinc Nutrition Consultative Group (IZINCG). Technical document \#1. Assessment of the risk of zinc deficiency in populations and options for its control. Food Nutr Bull 2004; 25: S91-204.

28. Meydani SN, Barnett JB, Dallal GE, Fine BC, Jacques PF, Leka LS, et al. Serum zinc and pneumonia in nursing home elderly. Am J Clin Nutr 2007; 86: 167-73.

29. Castillo O, Rozowski J, Cuevas A, Maiz A, Soto M, Mar- 
dones $\mathrm{F}$, et al. Ingesta de nutrientes en adultos mayores de la comuna de Providencia, Santiago de Chile. Rev Med Chile 2002; 130: 1335-42.

30. van Mierlo LA, Arends LR, Streppel MT, Zeegers MP, Kok FJ, Grobbee DE, et al. Blood pressure response to calcium supplementation: A meta-analysis of randomized controlled trials. J Hum Hypertens 2006; 20: 571-80.

31. Pittas AG, Lau J, Hu FB, Dawson-Hughes B. The role of vitamin $\mathrm{D}$ and calcium in type 2 diabetes. A systematic review and meta-analysis. J Clin Endocrinol Metab 2007; 92: 2017-29.

32. Hanley TM, Kiefer HL, Schnitzler AC, Marcello JE, Viglianti GA. Retinoid-dependent restriction of human immunodeficiency virus type 1 replication in monocytes/macrophages. J Virol 2004; 78: 2819-30.

33. Bjersing JL, Telemo E, Dahlgren U, Hanson LA. Loss of ileal IgA+ plasma cells and of CD4+ lymphocytes in ileal Peyer's patches of vitamin A deficient rats. Clin Exp Immunol 2002; 130: 404-8.

34. Stephensen CB, Jiang X, Freytag T. Vitamin A deficiency increases the in vivo development of IL-10-positive Th2 cells and decreases development of Th1 cells in mice. J Nutr 2004; 134: 2660-6.

35. Sánchez H, Albala C, Lera L, Castillo JL, Verdugo R, Lavados $\mathrm{M}$, et al. Comparison of two modes of vitamin
B12 supplementation on neuroconduction and cognitive function among older people living in Santiago, Chile: a cluster randomized controlled trial. A study protocol [ISRCTN 02694183]. Nutr J 2011; 27; 10: 100.

36. Dhonukshe-Rutten RAM, Pluijm SMF, De Groot LC, Lips P, Smit JH, Van Staveren WA. Homocysteine and vitamin B12 status relate to bone turnover markers, broadband ultrasound attenuation, and fractures in healthy elderly people. Journal of Bone and Mineral Research 2005; 20 (6): 921-9.

37. Tucker KL, Hannan MT, Qiao N, Jacques PF, Selhub J, Cupples LA, et al. Low plasma vitamin B12 is associated with lower BMD: The Framingham osteoporosis study. Journal of Bone and Mineral Research 2005; 20 (1): 1528.

38. Clarke R, Smith AD, Jobst KA, Refsum H, Sutton L, Ueland PM. Folic acid, vitamin B12 ad serum homocysteine levels in confirmed Alzheimer's disease. Arch Neurol 1998; 55: 1449-55.

39. Andres E, Loukili NH, Noel E, Kaltenbach G, Abdelgheni $\mathrm{MB}$, Perrin AE, et al. Vitamin B12 (cobalamin) deficiency in elderly patients. Cmaj 2004; 171 (3): 251-9.

40. Agarwal R. Vitamin B12 deficiency \& cognitive impairment in elderly population. Indian J Med Res 2011; 134 (4): 410-2. 